\title{
Diagnostic value of endobronchial ultrasonography for pulmonary tuberculosis
}

\author{
Shu-Min Lin, MD, Fu-Tsai Chung, MD, Chien-Da Huang, MD, Wen-Te Liu, MD, Chih-Hsia Kuo, MD, \\ Chun-Hua Wang, MD, Kang-Yun Lee, MD, PhD, Chien-Ying Liu, MD, Horng-Chyuan Lin, MD, \\ and Han-Pin Kuo, MD, PhD
}

Objectives: We sought to compare the diagnostic yields of acid-fast bacilli smears and Mycobacterium tubercu-
losis cultures in terms of bronchoalveolar lavage fluid and histologic examination of transbronchial lung biopsy
specimens for pulmonary tuberculosis by using bronchoscopy with versus without endobronchial ultrasonogra-
phy in patients with negative acid-fast bacilli smears or no sputum production.

Methods: From June 2005 to July 2006, a total of 451 patients were given diagnoses of and treated for pulmonary tuberculosis in a university-affiliated hospital. Among them, 121 patients who received bronchoscopy because of sputum-negative conditions were recruited. Of these, 73 patients received bronchoscopy with endobronchial ultrasonography, and 48 patients received conventional bronchoscopy.

Results: Patients who received bronchoscopy with endobronchial ultrasonography had higher diagnostic yields of acid-fast bacilli smears ( $31.5 \%$ vs $12.5 \%, P=.018)$ in bronchoalveolar lavage fluid, $M$ tuberculosis in bronchoalveolar lavage fluid $(67.1 \%$ vs $47.9 \%, P=.024)$, and pathologic reports of tuberculosis in transbronchial lung biopsy specimens $(32.9 \%$ vs $4.2 \%, P<.0001)$ than patients who received conventional bronchoscopy. With the aid of endobronchial ultrasonography, the overall diagnostic yield for tuberculosis by using bronchoscopic procedures (smears and cultures of bronchoalveolar lavage fluid and transbronchial lung biopsy specimens) was higher $(80.8 \%)$ than for those who did not undergo endobronchial ultrasonography $(58.3 \%, P=.035)$.

Conclusions: The addition of endobronchial ultrasonography to diagnostic bronchoscopy increased the sensitivity for proving the presence of tuberculosis in a population of patients with negative acid-fast bacilli smears or no sputum production.

Tuberculosis is the leading infectious cause of death among persons older than 5 years, particularly in Southeast Asia and sub-Saharan Africa. ${ }^{1}$ In the United States the incidence of tuberculosis more than doubled during the $1980 \mathrm{~s}^{2}{ }^{2}$ Sputum examination is the most useful method for diagnosing active pulmonary tuberculosis. However, about half of the patients suspected of active pulmonary tuberculosis are unable to produce sputum. ${ }^{3}$ Even if sputum is available, acid-fast bacilli (AFB) might not be found on repeated examination of direct smears. ${ }^{4}$ Therefore the condition of a negative sputum smear and no sputum production is one of the causes of delay in tuberculosis diagnosis. Delays in diagnosis and treatment have been recognized as an important cause of death from pulmonary tuberculosis. ${ }^{2,5}$ Patients might be left untreated until Mycobacterium tuberculosis grows from the culture specimens. Untreated patients with pulmonary tuberculosis can

\footnotetext{
From the Department of Thoracic Medicine, Chang Gung Memorial Hospital, Chang Gung University, School of Medicine, Taipei, Taiwan.

Supported by NSC 97-2314-B-182A-088-MY3, National Science Council, Taiwan, ROC.

Received for publication Nov 21, 2008; revisions received March 5, 2009; accepted for publication April 1, 2009.

Address for reprints: Han-Pin Kuo, MD, PhD, Department of Thoracic Medicine, Chang Gung Memorial Hospital, 199 Tun-Hwa N Rd, Taipei, Taiwan (E-mail: q8828@ms11.hinet.net).

J Thorac Cardiovasc Surg 2009;138:179-84

$0022-5223 / \$ 36.00$

Copyright (C) 2009 by The American Association for Thoracic Surgery

doi:10.1016/j.jtcvs.2009.04.004
}

constitute an important reservoir for disease transmission in the community. Possible approaches to deal with this condition include empiric treatment with antituberculosis medications, close follow-up of the progression of chest radiographic lesions for 6 to 8 weeks while waiting for culture results, and alternative methods for obtaining lower respiratory tract specimens. ${ }^{4,6}$ However, the first 2 approaches might delay the true diagnosis. In contrast, invasive procedures, such as percutaneous lung biopsy, carry the potential risk for hemorrhage and pneumothorax, which are especially hazardous in patients with poor lung reserves. Thus bronchoscopy can be used in this condition to provide the clue for earlier diagnosis of tuberculosis or to obtain specimens in patients without spontaneous sputum.

Bronchoscopy with AFB smears and cultures for $M$ tuberculosis of bronchoalveolar lavage fluid (BALF) and histologic examination of transbronchial lung biopsy (TBLB) specimens have been introduced to reach an early bacteriologic diagnosis of tuberculosis in sputum-negative patients or patients unable to expectorate, with varying results. ${ }^{3,6-8}$ Endobronchial ultrasonography (EBUS), a newly introduced technique, has been applied to the assessment of peripheral lung lesions. ${ }^{9,10}$ Compared with conventional bronchoscopy, EBUS offers the benefits of visualizing the parabronchial structure, confirming the precise location of peripheral lung lesions and therefore improving the diagnostic yield. ${ }^{11,12}$ By means of demonstration of a good correlation between 


\section{Abbreviations and Acronyms \\ $\mathrm{BALF}=$ bronchoalveolar lavage fluid \\ $\mathrm{CT}=$ computed tomography \\ EBUS $=$ endobronchial ultrasonography \\ $\mathrm{TBLB}=$ transbronchial lung biopsy}

EBUS images and histology of lung lesions, the characteristics of EBUS images also provide useful clues in differentiating malignant and benign lesions in the peripheral lung. ${ }^{11}$ However, the role of bronchoscopy with the aid of EBUS in the diagnosis of pulmonary tuberculosis is still unclear.

The primary aim of this study was to compare the diagnostic yields of AFB smears, $M$ tuberculosis cultures in BALF, and histologic examination of TBLB specimens for pulmonary tuberculosis by using bronchoscopy with or without EBUS in patients with negative AFB smears or no sputum production. The secondary aim was to determine the contributions of EBUS-guided procedures on early diagnosis of pulmonary tuberculosis.

\section{MATERIALS AND METHODS \\ Study Subjects}

The study retrospectively analyzed patients who underwent bronchoscopy at Chang Gung Memorial Hospital, a university-affiliated, 3300-bed hospital in Taiwan. From June 2005 to July 2006, a total 451 patients were given diagnoses of and treated for pulmonary tuberculosis in the hospital. Among them, 121 patients received bronchoscopy because of a lack of spontaneous sputum or negative AFB smears in 3 consecutive daily sputum specimens. Of these, 73 who received bronchoscopic examination with EBUS guidance were defined as the EBUS group. The other 48 patients underwent bronchoscopic examinations during the time period when EBUS was not available. They received bronchoscopic examination without EBUS guidance and were designated as the non-EBUS group. All the patients received bronchoscopy-related procedures for the diagnosis of active pulmonary tuberculosis, including AFB smears and cultures for $M$ tuberculosis from BALF and histologic examination of TBLB specimens. No patients were infected with HIV. All patients then provided written informed consent to undergo bronchoscopy. This study was approved by the hospital's internal review board. Patients' baseline characteristics included age, sex, location and size of lesion, and radiologic appearance. Underlying medical history was also collected, including histories of diabetes mellitus, hypertension, malignancy, chronic airway obstruction disease, and previous tuberculosis infection.

\section{Bronchoscopic Procedures and Equipment}

The patients were referred to the institutional bronchoscopic center for procedures. The bronchoscopic examinations were performed by 2 experienced independent bronchoscopists (S. M. Lin and F. T. Chung), each of whom had performed more than 200 bronchoscopic procedures each year. The EBUS group was retrieved after each of the bronchoscopists had performed more than 50 EBUS procedures to ensure the quality of EBUS in the study. The probable location of the lesion was determined initially by using traditional posterior anterior chest radiography with or without chest computed tomography (CT). Both groups had similar percentages of patients who received a CT scan before bronchoscopy (EBUS vs nonEBUS, $27 / 73[37 \%]$ vs 20/48 [41.7\%], respectively; $P=.704)$. A flexible fiberoptic bronchoscope (BF-P240 or BF-40; Olympus, Tokyo, Japan) was then inserted through the nostril after local spray of $2 \%$ lidocaine for anesthesia. The heart rate and oxygen saturation of each patient were monitored by using a pulse oximeter during the procedure. In the EBUS group a 20$\mathrm{MHz}$, radial, mechanical-type ultrasonographic probe (model UM-S20S20R, Olympus) and an ultrasound unit (Endoscopic Ultrasound System, Olympus) were used in the procedure. The EBUS catheter was advanced into the fourth- to sixth-order bronchi to assess lesions in the periphery of the lung. Once the lesion was located by using EBUS, the neighbor bronchiolar orifices were also examined to ensure the precise location of lesions. Each EBUS image was evaluated for 3 features, including the continuous margin of the lesion, the presence of an air bronchogram, and the heterogeneous echogenicity of the internal structure. ${ }^{11}$ The EBUS procedure was performed without a guide sheath.

Bronchoalveolar lavage was performed by means of infusion of 6 aliquots of $50 \mathrm{~mL}$ of normal saline, and the TBLB specimen was taken last. ${ }^{13}$ The return was submitted for AFB smear and culture. Lavaged specimens were processed with standard methodology and stained with fluorochrome. ${ }^{14}$ Positive smears were confirmed by means of Ziehl-Neelsen staining. Specimens were cultured with a Bactec $12 \mathrm{~B}$ vial (radiometric Bactec 460-TB system; Becton Dickinson, Franklin Lakes, NJ) and Lowenstein-Jensen media (Bio-Rad, Marnesla-Coquette, France). TBLB was performed for lung parenchyma biopsy specimens. At least 3 pieces of tissue specimens were obtained by means of TBLB. The specimens were placed in $10 \%$ buffered formaldehyde solution and subsequently examined with hematoxylin and eosin and Ziehl-Neelsen staining. The overall mean time for the procedure was $10.5 \pm 2.1$ minutes for the EBUS group and 9.3 \pm 2.3 minutes for the non-EBUS group.

\section{Diagnosis of Pulmonary Tuberculosis}

In patients who are suspected of having tuberculosis for radiologic reasons, the diagnosis of tuberculosis was made based on positive $M$ tuberculosis cultures in specimens or histologic demonstration of granulomatous inflammation with Langham's giant cells and caseation. The final diagnosis of active pulmonary tuberculosis was reconfirmed based on consensus between at least 2 respiratory specialists after at least 3 months of patient follow-up. Ambiguities were resolved by means of independent review with another chest physician or radiologist. Patients with a positive AFB smear in BALF but a negative result on $M$ tuberculosis culture were excluded $(\mathrm{n}=2)$.

\section{Statistical Analysis}

Data are expressed as either group percentages (categorical variables) or means \pm standard deviations (continuous variables). Data were compared between the EBUS and non-EBUS groups. Categorical variables were compared by using the $\chi^{2}$ test (or the Fisher's exact test when the expected number of any cells was $\leq 5$ ). We applied the $t$ test to compare continuous variables with normal distribution and the Mann-Whitney test for continuous variables not distributed normally. All analyses were performed with SPSS software version 10.0 (SPSS, Inc, Chicago, Ill).

\section{RESULTS \\ Baselines}

The baseline characteristics of the enrolled patients are summarized in Table 1 . There were no significant differences between groups regarding demographic variables, size of the lesion, location of the lesion, radiologic appearance, and chronic comorbidities. For the EBUS group, 61 (83.6\%) of 73 lesions were localized by means of EBUS. The presence of an air bronchogram was observed in $54(74 \%)$ of 73 patients; lesions with continuous margins and homogenous echogenicity were detected in $41(56.1 \%)$ of 73 and 53 
TABLE 1. Characteristics of patients with pulmonary tuberculosis

\begin{tabular}{|c|c|c|c|}
\hline Baseline characteristics & Without EBUS $(n=48)$ & With EBUS $(n=73)$ & $P$ value \\
\hline Age $(y)$, mean $\pm S D$ & $48.5 \pm 22.8$ & $56.0 \pm 14.5$ & .103 \\
\hline Female sex & $22(45.3 \%)$ & $22(30.1 \%)$ & .086 \\
\hline Size of lesion $(\mathrm{cm})$, mean $\pm \mathrm{SD}$ & $3.4 \pm 1.4$ & $3.8 \pm 1.6$ & .225 \\
\hline Lesion size $<3 \mathrm{~cm}$ & $22(45.3 \%)$ & $31(42.5 \%)$ & .852 \\
\hline Lesion localized by EBUS & NA & $61(83.6 \%)$ & NA \\
\hline Lesion $(<3 \mathrm{~cm})$ localized by EBUS & NA & $23(74.2 \%)$ & NA \\
\hline \multicolumn{4}{|l|}{ Location of lesion } \\
\hline Right upper lobe & $22(45.3 \%)$ & $24(32.9 \%)$ & .182 \\
\hline Right middle lobe & $4(8.4 \%)$ & $11(15.1 \%)$ & .399 \\
\hline Right lower lobe & $0(0 \%)$ & $2(2.7 \%)$ & .278 \\
\hline Left upper lobe & $18(37.5 \%)$ & $20(27.4 \%)$ & .317 \\
\hline Left lower lobe & $4(8.4 \%)$ & $16(21.9 \%)$ & .078 \\
\hline \multicolumn{4}{|l|}{ Radiologic appearance } \\
\hline With cavity & $6(12.5 \%)$ & $10(13.7 \%)$ & 1.00 \\
\hline With lymphadenopathy & $7(14.6 \%)$ & $12(16.4 \%)$ & 1.00 \\
\hline Fibronodular infiltration & $26(54.2 \%)$ & $44(60.3 \%)$ & .574 \\
\hline Alveolar consolidation & $14(29.2 \%)$ & $23(31.5 \%)$ & .842 \\
\hline Diffuse nodule or mass & $4(8.4 \%)$ & $6(8.2 \%)$ & 1.00 \\
\hline \multicolumn{4}{|l|}{ Echoic features by EBUS } \\
\hline Presence of air bronchogram & NA & $54(74.0 \%)$ & NA \\
\hline Continuous margin & NA & $41(56.1 \%)$ & NA \\
\hline Homogenous echogenicity & NA & $53(72.5 \%)$ & NA \\
\hline \multicolumn{4}{|l|}{ Chronic coexisting condition } \\
\hline Diabetes mellitus & $8(16.7 \%)$ & $20(27.4 \%)$ & .193 \\
\hline Hypertension & $6(12.5 \%)$ & $12(16.4 \%)$ & .611 \\
\hline Chronic renal insufficiency & $2(4.2 \%)$ & $7(9.6 \%)$ & .316 \\
\hline Cancer & $1(2.1 \%)$ & $4(5.5 \%)$ & .647 \\
\hline Chronic obstructive pulmonary disease & $3(6.3 \%)$ & $4(5.5 \%)$ & 1.00 \\
\hline Previous tuberculosis infection & $2(4.2 \%)$ & $4(5.5 \%)$ & 1.00 \\
\hline
\end{tabular}

Values are presented as numbers (percentages), where shown. EBUS, Endobronchial ultrasonography; $S D$, standard deviation; $N A$, not available.

$(72.5 \%)$ of 73 patients, respectively. Diabetes mellitus was the most frequent chronic comorbidity in both groups. There were no cancers diagnosed in the study population.

\section{Yields of Sputum and Lavaged Specimens for Tuberculosis Diagnosis}

Thirty-six (49.3\%) of 73 patients in the EBUS group and $18(37.5 \%)$ of 48 patients undergoing conventional bronchoscopy had no spontaneous sputum, whereas 37 $(50.7 \%)$ of 73 patients in the EBUS group and $30(62.5 \%)$ of 48 patients undergoing conventional bronchoscopy had negative AFB smears in 3 consecutive daily sputum specimens. The diagnostic yields of AFB smears in BALF (31.5\% [23/73], $P=.018), M$ tuberculosis in BALF (67.1\% [49/73], $P=.04)$, and histologic diagnosis of tuberculosis by means of TBLB (32.9\% [24/73], $P<.001)$ were significantly higher in patients undergoing EBUS. There were $12.5 \%$ (6/48), 47.9\% (23/48), and 4.2\% (2/48) patients who underwent conventional bronchoscopy, respectively (Table 2). With the aid of EBUS, the overall diagnostic yield for tuberculosis by means of bronchoscopic procedures (smears and cultures of BALF and TBLB specimens) was higher $(80.8 \%)$ than that for those who did not undergo EBUS (58.3\%, $P=.035$; Table 2).

\section{Yields of Lavaged Specimens for Tuberculosis Diagnosis Stratified by Size of Lesion}

In patients with lesions smaller than $3 \mathrm{~cm}$ (Table 3), bronchoscopy with EBUS $(\mathrm{n}=31)$ produced higher diagnostic yields of AFB smears in BALF $(35.5 \%$ vs $4.5 \%$,

TABLE 2. Yield of sputum and EBUS-guided bronchial lavage smear, culture, and biopsy results for the diagnosis of tuberculosis

\begin{tabular}{lccc}
\hline \multicolumn{1}{c}{ Result } & Without EBUS* & With EBUS* & P value \\
\hline Positive sputum culture & $18 / 30(60 \%)$ & $12 / 37(32.4 \%)$ & .029 \\
Positive BALF smear & $6 / 48(12.5 \%)$ & $23 / 73(31.5 \%)$ & .018 \\
Positive BALF culture & $23 / 48(47.9 \%)$ & $49 / 73(67.1 \%)$ & .040 \\
Positive TBLB & $2 / 48(4.2 \%)$ & $24 / 73(32.9 \%)$ & $<.0001$ \\
Positive BALF smear, culture, or TBLB specimen & $29 / 48(60.4 \%)$ & $59 / 73(80.8 \%)$ & .020 \\
\hline
\end{tabular}

EBUS, Endobronchial ultrasonography; $B A L F$, bronchoalveolar lavage fluid; $T B L B$, transbronchial lung biopsy. *Positive sample number/total sample number. 
TABLE 3. Yield of EBUS-guided bronchial lavage smear, culture, and biopsy results for the diagnosis of tuberculosis based on the size of the lesion

\begin{tabular}{|c|c|c|c|c|c|c|}
\hline & \multicolumn{2}{|c|}{ Lesion $<3 \mathrm{~cm}$} & \multirow[b]{2}{*}{$P$ value } & \multicolumn{2}{|c|}{ Lesion $>3 \mathrm{~cm}$} & \multirow[b]{2}{*}{$P$ value } \\
\hline & $\begin{array}{c}\text { Without EBUS } \\
\quad(n=22)\end{array}$ & $\begin{array}{c}\text { With EBUS } \\
(\mathbf{n}=\mathbf{3 1})\end{array}$ & & $\begin{array}{c}\text { Without EBUS } \\
\quad(n=26) \\
\end{array}$ & $\begin{array}{c}\text { With EBUS } \\
(\mathrm{n}=\mathbf{4 2})\end{array}$ & \\
\hline Positive BALF smear & $1(4.5 \%)$ & $11(35.5 \%)$ & .009 & $5(22.7 \%)$ & $12(28.6 \%)$ & .565 \\
\hline Positive BALF culture & $6(27.3 \%)$ & $19(61.3 \%)$ & .025 & $17(77.3 \%)$ & $30(71.4 \%)$ & .602 \\
\hline Positive TBLB & $1(4.5 \%)$ & $10(32.3 \%)$ & .017 & $1(4.5 \%)$ & $14(33.3 \%)$ & .006 \\
\hline Positive BALF smear, culture, or & $7(31.8 \%)$ & $25(80.6 \%)$ & .0005 & $21(80.7 \%)$ & $24(80.9 \%)$ & 1.00 \\
\hline
\end{tabular}

TBLB specimen

EBUS, Endobronchial ultrasonography; $B A L F$, bronchoalveolar lavage fluid; $T B L B$, transbronchial lung biopsy.

$P=.009)$, M tuberculosis in BALF $(61.3 \%$ vs $27.3 \%, P=$ $.025)$, and pathologic reports of tuberculosis diagnosis in TBLB specimens $(32.3 \%$ vs $4.5 \%, P=.017)$ than conventional bronchoscopy $(n=22)$. With the aid of EBUS, the overall diagnostic yield for tuberculosis by using bronchoscopic procedures was higher $(80.6 \%)$ than that for those who did not undergo EBUS $(31.8 \%, P=.0005)$. In patients with lesions larger than $3 \mathrm{~cm}$ in size, bronchoscopy with EBUS $(n=42)$ produced higher diagnostic yields of pathologic reports of tuberculosis in TBLB specimens (33.3\% vs $4.5 \%, P=.006$ ) but similar yields of AFB smears in BALF (28.6\% vs $22.7 \%, P=.565), M$ tuberculosis in BALF (71.4\% vs $77.3 \%, P=.602)$, and overall diagnosis for tuberculosis $(80.9 \%$ vs $80.7 \%, P=1.00)$ by using bronchoscopic procedures compared with conventional bronchoscopy $(n=26)$.

\section{First Results for Diagnosis of Pulmonary Tuberculosis}

Table 4 summarizes the first available results for initial diagnosis of pulmonary tuberculosis in the sputum-negative patients. There was a higher proportion of patients in the EBUS group initially documented to have pulmonary tuberculosis based on positive AFB smears of BALF (31.5\% [n $=23], P=.018)$ and histologic diagnosis of tuberculosis by using TBLB $(24.7 \%[\mathrm{n}=18], P=.003)$ when compared with those in the conventional bronchoscopy group (12.5\% $[n=6]$ and $4.2 \%[n=2]$, respectively; Figure 1). There was a higher proportion of patients in the conventional group with tuberculosis diagnosis documented only by positive culture $(70.8 \%$, either from BALF or sputum) than among patients undergoing EBUS $(27.4 \%, P<.001$; Table 3; Figure 1). With the aid of EBUS, the first available results from bronchoscopy-guided procedures documented tuberculosis in 59 (23 patients based on BALF smears, 18 patients based on BALF cultures, and 18 patients based on TBLB specimens) of 73 patients, with only 28 (6 patients based on BALF smears, 20 patients based on BALF cultures, and 2 patients based on TBLB specimens) of 48 patients undergoing conventional bronchoscopy $(80.8 \%$ vs $58.3 \%, P=$ $.035)$. For those patients with negative results with bronchoscopic procedures, $2(2.7 \%)$ of the 73 patients undergoing EBUS and $14(29.2 \%)$ of 48 patients not undergoing EBUS were given diagnoses based on positive sputum $M$ tuberculosis cultures. CT-guided biopsy was required for the diagnosis of tuberculosis in $6(12.5 \%)$ of 48 patients in the conventional group and $12(16.4 \%)$ of 72 patients in the EBUS group.

\section{Treatment of Pulmonary Tuberculosis}

The time to diagnosis of tuberculosis in patients undergoing EBUS (23.1 \pm 16.7 days) was shorter than that in patients undergoing conventional bronchoscopy (32.4 \pm 25.7 days, $P=.017)$. Similarly, the time to initiate treatment in patients receiving conventional bronchoscopy (39.7 \pm 25.8 days) was longer than that in patients receiving bronchoscopy with EBUS $(29.4 \pm 17.1$ days, $P=.009)$. However, the EBUS group (58/73 [79\%]) had a similar rate of complete tuberculosis treatment when compared with the conventional group $(35 / 48[73 \%], P=.404)$.

TABLE 4. The first available study results for clinical diagnosis of pulmonary tuberculosis

\begin{tabular}{|c|c|c|c|}
\hline Diagnosis established based on: & Without EBUS $(n=48)$ & With EBUS $(n=73)$ & $P$ value \\
\hline Positive BALF smear & $6(12.5 \%)$ & $23(31.5 \%)$ & .018 \\
\hline Positive BALF and sputum culture & $4(8.3 \%)$ & $4(5.5 \%)$ & .711 \\
\hline Positive BALF but negative sputum culture & $16(33.3 \%)$ & $14(21.9 \%)$ & .089 \\
\hline Positive sputum but negative BALF culture & $14(29.2 \%)$ & $2(2.7 \%)$ & $<.0001$ \\
\hline Positive TBLB specimen & $2(4.2 \%)$ & $18(24.7 \%)$ & .003 \\
\hline CT-guided biopsy & $6(12.5 \%)$ & $12(16.4 \%)$ & .611 \\
\hline Positive BALF smear or TBLB specimen & $8(16.7 \%)$ & $41(56.2 \%)$ & $<.0001$ \\
\hline Only culture positive & $34(70.8 \%)$ & $20(27.4 \%)$ & $<.0001$ \\
\hline Positive lavage smear, culture, or biopsy specimen & $28(58.3 \%)$ & $59(80.8 \%)$ & .035 \\
\hline
\end{tabular}

$E B U S$, Endobronchial ultrasonography; $B A L F$, bronchoalveolar lavage fluid; $T B L B$, transbronchial lung biopsy; $C T$, computed tomography. 


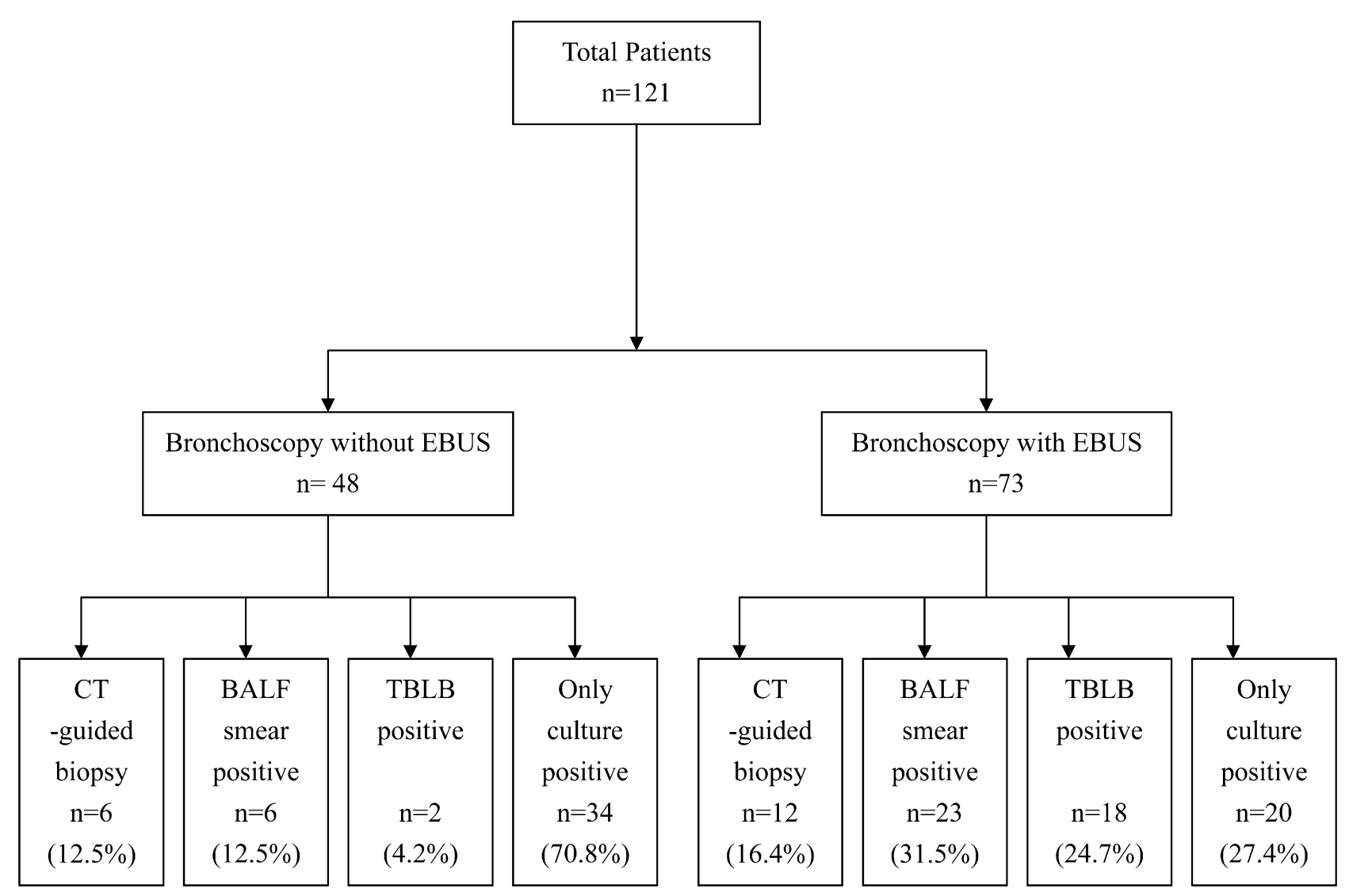

FIGURE 1. Analysis of the first available results for diagnosis of pulmonary tuberculosis. EBUS, Endobronchial ultrasonography; $C T$, computed tomography; $B A L F$, bronchoalveolar lavage fluid; $T B L B$, transbronchial lung biopsy.

\section{Complications}

Pneumothorax occurred in $1(1.0 \%)$ patient after the EBUS procedure and resolved spontaneously, but no pneumothorax developed in the conventional bronchoscopy group. No major bleeding with hemodynamic instability developed during the procedure in either group.

\section{DISCUSSION}

In patients who are suspected of having tuberculosis for radiologic reasons but with negative sputum AFB smears or no sputum production, the introduction of EBUS has been shown in this report to increase the diagnostic yields for tuberculosis by bronchoscopy-related procedures, including AFB smears and $M$ tuberculosis cultures from BALF, and pathologic documentation by TBLB. Combining AFB smears of BALF and pathology by TBLB, bronchoscopy with EBUS could achieve a higher rate of rapid diagnosis of tuberculosis than conventional bronchoscopy.

The negative sputum smear and a lack of spontaneous sputum in patients with pulmonary tuberculosis have been reported to be the major causes of treatment delay for tuberculosis. ${ }^{16}$ Fiberoptic bronchoscopy has been used for obtaining lower respiratory tract sections and lung tissue in those patients to avoid a delay in treatment. ${ }^{3}$ The diagnostic yield for pulmonary tuberculosis of BALF in patients with negative sputum smears has been demonstrated with varying results, ranging from $7.5 \%$ to $24 \%$ for smears and $15 \%$ to $67.4 \%$ for cultures. ${ }^{3,6-8}$ The wide range of variability in conventional bronchoscopy might be due to the lesions that could not be seen with conventional bronchoscopy. ${ }^{17}$ Once the lesion is not visible with conventional bronchoscopy, bronchoalveolar lavage and TBLB are performed in a bestguess fashion or with the aid of a fluoroscope. ${ }^{18}$ In the present study our diagnostic yield of conventional bronchoscopy for pulmonary tuberculosis $(58.3 \%)$ was in line with that seen in previous reports. In the lesions that are not visualized with conventional bronchoscopy, EBUS images allow confirmation of the precise location of lesions by means of direct visualization. Therefore with the aid of EBUS to accurately localize the lesion, bronchoscopy-related procedures, especially bronchoalveolar lavage and TBLB, increased the overall diagnostic yield for pulmonary tuberculosis to more than $80 \%$. In addition, the use of EBUS during bronchoscopic procedures did not increase the risk for pneumothorax. The incidence of pneumothorax in our study is in line with that seen in previous reports, approximately $1 \% .^{19,20}$

The image characteristics of EBUS provide clues in determining the nature of peripheral abnormalities, such as 
atelectasis, tumor, and inflammation, based on their different ultrasonic features. ${ }^{21}$ A previous report has correlated histopathologic findings from surgical specimens to develop a classification system of sonographic images to distinguish between benign and malignant lesions. ${ }^{21}$ Our previous study also indicates that with 3 image patterns, including the presence of continuous margin, absence of a linear discrete air bronchogram, and heterogenous echogenicity of the internal structure, EBUS provides a useful tool for differentiating benign lesions from malignancy in peripheral lung lesions. ${ }^{11}$ In the present study the EBUS image pattern of pulmonary tuberculosis showed a high percentage of benign characteristic features, including the presence of an air bronchogram and homogenous echogenicity. Thus apart from its role in improving the diagnostic yield for pulmonary tuberculosis, the EBUS image pattern of pulmonary tuberculosis lesions might offer clues for differentiating benign lesions from malignant peripheral lung lesions.

Rapid diagnosis and treatment have long been considered a crucial factor for better outcome of patients with tuberculosis. ${ }^{2,5,15,16}$ In this study AFB smears of BALF and pathology by TBLB specimens offered rapid evidence for tuberculosis infection in more $50 \%$ of the patients in the EBUS group but only $16.7 \%$ of the patients receiving conventional bronchoscopy. The high diagnostic yield of EBUS-guided bronchoscopy for early diagnosis of tuberculosis infection might therefore avoid a delay in administration of antituberculosis treatment or the need for further invasive procedures, such as CT-guided or surgical biopsy, to reach a definite diagnosis. However, a false-positive result on a BALF smear can occur, and therefore $M$ tuberculosis culture in BALF is still required for a definite diagnosis.

Several limitations of this study warrant mention. First, this is a retrospective study with all the patients with pulmonary tuberculosis, and therefore the sensitivity, specificity, and predictive value cannot be determined. In addition, the study recruited patients with negative AFB smears or no sputum production. Therefore the results of our study could not be extended to all the patients with suspicious tuberculosis lesions.

In conclusion, sputum AFB smears and $M$ tuberculosis cultures should be first performed in patients under suspicion of pulmonary tuberculosis. Once bronchoscopy is arranged to obtain lower airway secretion for documentation of pulmonary tuberculosis in patients with negative sputum smears or no sputum production, EBUS-guided bronchoscopic procedures can provide higher diagnostic yield and rapid diagnosis.

\section{References}

1. Raviglione MC, Snider DE Jr, Kochi A. Global epidemiology of tuberculosis. Morbidity and mortality of a worldwide epidemic. JAMA. 1995;273: 220-6.

2. Pablos-Mendez A, Sterling TR, Frieden TR. The relationship between delayed or incomplete treatment and all-cause mortality in patients with tuberculosis. JAMA. 1996;276:1223-8.

3. Chawla R, Pant K, Jaggi OP, Chandrashekhar S, Thukral SS. Fibreoptic bronchoscopy in smear-negative pulmonary tuberculosis. Eur Respir J. 1988;1: 804-6.

4. Anonymous. Sputum-smear-negative pulmonary tuberculosis: controlled trial of 3-month and 2-month regimens of chemotherapy. Lancet. 1979;1:1361-3.

5. Enarson DA, Grzybowski S, Dorken E. Failure of diagnosis as a factor in tuberculosis mortality. CMAJ. 1978;118:1520-2.

6. Willcox PA, Benatar SR, Potgieter PD. Use of the flexible fibreoptic bronchoscope in diagnosis of sputum-negative pulmonary tuberculosis. Thorax. 1982; 37:598-601.

7. Brown M, Varia H, Bassett P, Davidson RN, Wall R, Pasvol G. Prospective study of sputum induction, gastric washing, and bronchoalveolar lavage for the diagnosis of pulmonary tuberculosis in patients who are unable to expectorate. Clin Infect Dis. 2007; 44:1415-20.

8. Charoenratanakul S, Dejsomritrutai W, Chaiprasert A. Diagnostic role of fiberoptic bronchoscopy in suspected smear negative pulmonary tuberculosis. Respir Med. 1995;89:621-3.

9. Hurter T, Hanrath P. Endobronchial sonography: feasibility and preliminary results. Thorax. 1992;47:565-7.

10. Kurimoto N, Murayama M, Yoshioka S, Nishisaka T. Analysis of the internal structure of peripheral pulmonary lesions using endobronchial ultrasonography. Chest. 2002;122:1887-94.

11. Kuo CH, Lin SM, Chen HC, Chou CL, Yu CT, Kuo HP. Diagnosis of peripheral lung cancer with three echoic features via endobronchial ultrasound. Chest. 2007; 132:922-9.

12. Yang MC, Liu WT, Wang CH, Lin HC, Chen HC, Chou CL, et al. Diagnostic value of endobronchial ultrasound-guided transbronchial lung biopsy in peripheral lung cancers. J Formos Med Assoc. 2004;103:124-9.

13. Wang CH, Liu CY, Wan YL, Chou CL, Huang KH, Lin HC, et al. Persistence of lung inflammation and lung cytokines with high-resolution $\mathrm{CT}$ abnormalities during recovery from SARS. Respir Res. 2005;6:42.

14. McWilliams T, Wells AU, Harrison AC, Lindstrom S, Cameron RJ, Foskin E. Induced sputum and bronchoscopy in the diagnosis of pulmonary tuberculosis. Thorax. 2002;57:1010-4.

15. Lim TK, Mukhopadhyay A, Gough A, Khoo KL, Khoo SM, Lee KH, et al. Role of clinical judgment in the application of a nucleic acid amplification test for the rapid diagnosis of pulmonary tuberculosis. Chest. 2003;124:902-8.

16. Mathur P, Sacks L, Auten G, Sall R, Levy C, Gordin F. Delayed diagnosis of pulmonary tuberculosis in city hospitals. Arch Intern Med. 1994;154:306-10.

17. Fielding DI, Robinson PJ, Kurimoto N. Biopsy site selection for endobronchial ultrasound guide-sheath transbronchial biopsy of peripheral lung lesions. Intern Med J. 2008;38:77-84

18. Yoshikawa M, Sukoh N, Yamazaki K, Kanazawa K, Fukumoto S, Harada M, et al. Diagnostic value of endobronchial ultrasonography with a guide sheath for peripheral pulmonary lesions without X-ray fluoroscopy. Chest. 2007;131: 1788-93.

19. Kurimoto N, Miyazawa T, Okimasa S, Maeda A, Oiwa H, Miyazu Y, et al. Endobronchial ultrasonography using a guide sheath increases the ability to diagnose peripheral pulmonary lesions endoscopically. Chest. 2004;126:959-65.

20. Herth FJ, Eberhardt R, Becker HD, Ernst A. Endobronchial ultrasound-guided transbronchial lung biopsy in fluoroscopically invisible solitary pulmonary nodules: a prospective trial. Chest. 2006;129:147-50.

21. Omori S, Takiguchi Y, Hiroshima K, Tanabe N, Tatsumi K, Kimura H, et al. Peripheral pulmonary diseases: evaluation with endobronchial US initial experience. Radiology. 2002;224:603-8. 